\title{
Factores de riesgo asociados al bajo peso al nacer
}

\author{
HUGO SALINAS(1), MARCIA ERAZO(2), CAROLINA PASTENE(1), \\ ALVARO REYES(2), JORGE CATALÁN(1), SERGIO CARMONA(1) et al.
}

\section{RESUMEN}

En Chile, existe un porcentaje de niños con bajo peso al nacer, cifra muy similar a las tasas de países desarrollados. Estos presentan mayor riesgo de déficit de desarrollo neurológico, metabólico y capacidad cognitiva, requiriendo además de costosos cuidados especiales a lo largo de su desarrollo. El presente trabajo busca estimar los factores de riesgo para bajo peso al nacer, con el fin de detectarlos a tiempo y evitar asi su ocurrencia. Nuestro estudio consideró a los niños nacidos vivos con pesos menores a 2.500 gramos $(n=334)$, en la Maternidad del Hospital Clínico de la Universidad de Chile entre los años 2001 y 2002. La información se obtuvo durante la atención prenatal y el parto, se compararon los niños con muy bajo peso (<2.000 gramos) con aquéllos que pesaron 2.000 gramos y más. El análisis estadístico consideró la evaluación de la calidad y consistencia de los datos obtenidos, para esto se empleó el programa Stata 8.0. Se observó que el factor de riesgo mayormente asociado a muy bajo peso al nacer es la prematurez $(89 \%)$, seguido por la edad materna extrema (50,7\%) y, en tercer lugar, la preeclampsia $(44,5 \%)$. Este trabajo concluye que los factores de riesgo de muy bajo peso al nacer han variado en la última década. Esto explicado por el cambio en el perfil epidemiológico que la población chilena experimentó en estos últimos años, transformando los problemas de malmutrición por déficit por los de exceso y los programas ministeriales que centraron sus esfuerzos en las poblaciones de riesgo nutricional, reduciendo así la malnutrición por déficit. El bajo peso de un niño prematuro es explicable por la ganancia de peso de entre 200 y 300 gramos en promedio por cada semana de permanencia dentro del útero, especialmente hacia el final de la gestación. Con respecto a la edad materna extrema, se puede afirmar que las mujeres adolescentes presentan un mayor riesgo de parto prematuro o de recién nacido de término con bajo peso debido a un estado nutricional pre concepcional deficitario (escasa ganancia de peso durante el embarazo y malos controles). En pacientes mayores de 35 años, se plantea que la existencia de patología crónica (diabetes, hipertensión, etc) motivaria adelantar el parto. La preeclampsia es un factor de riesgo conocido de bajo peso de nacimiento, que ocasiona alteraciones de la placentación. Lo anterior orienta a considerar que es posible prevenir la aparición de estos factores y así disminuir el porcentaje de niños con pesos muy bajos de nacimiento.

Palabras clave: Bajo peso, preeclampsia, edad materna, prematurez.

\footnotetext{
(1) Departamento de Obstetricia y Ginecología. Hospital Clínico de la Universidad de Chile. hsalinas@ns.hospital.uchile.cl

(2) Alumno Postgrado. Escuela de Salud Pública. Facultad de Medicina. Universidad de Chile.
} 


\section{RISK FACTORS ASSOCIATED TO LOW BIRTH WEIGHT}

In Chile there exists a percentage of children with low weight at birth which is very similar to the rate in developed countries. These children are in greater risk of neurologic, metabolic and cognitive capacity development deficit, also requiring special care during their development. The present paper seeks to appraise the risk factors of low weight at birth, so as to detect them in time and thus avoid their occurrence. Our study was centered on live-born babies with birth weights below 2.500 grams $(n=334)$, in the Maternity of the University of Chile Clinical Hospital during the years 2001 and 2002. The information was obtained during prenatal attention and delivery; newborns with very low birth weight $(<2.000$ grams) were compared with those weighing 2000 grams or more. The statistical analysis considered the evaluation of the quality and consistency of the data obtained, for which purpose the Stata 8.0 program was used. It was observed that the main factor associated with very low birth weight is prematurity (89\%), followed by extreme age of the mother (50.7\%) and, in the third place, preeclampsia (44.5\%). This study concludes that the risk factors of very low weight at birth have varied in the last decade. This is explained by the change in the epidemiologic profile which the Chilean population has experienced during the last years, changing the problems of malnutrition due to deficit for those of excess and the ministerial programs that centered their efforts on the populations in nutritional risk, thus reducing malnutrition due to deficit. The low birth weight of a premature child can be explained by the gain in weight of between 200 and 300 grams as an average for each week of permanence within the uterus, specially toward the end of gestation. As respects the extreme age of the mother, it can be confirmed that adolescent girls present a greater risk of premature delivery or low weight fullterm delivery due to a pre-conceptional deficitary nutritional state (low weight gain during pregnancy and bad controls). In women of over 35 years of age, the point is raised that the existence of chronic pathology (diabetes, hypertension, etc.) would provide the grounds for an earlier delivery. Preecclampsia is a known risk factor for low weight at birth, which produces alterations of placentation. This leads us to consider that it is possible to forestall the appearance of these factors and thus diminish the number of infants with very low birth weights.

Key words: Low weight, preeclampsia, mother's age, prematurity.

\section{INTRODUCCIÓN}

En Chile, el peso al nacer ha tenido una evolución favorable en los últimos años, ya que -por un lado- el promedio de peso ha ido incrementando hasta llegar a cifras cercanas a 3.500 gramos y, por otro, el bajo peso al nacer se encuentra en una proporción semejante a la de países desarrollados $(4,2 \%)^{\prime}$.

No obstante este avance, aún persisten niños que nacen con pesos muy bajos (peso inferior a 2.000 gramos), quienes han sido considerados como los de mayor riesgo debido a que tendrían un menor desarrollo neurológico, habilidades cognitivas y problemas metabólicos ${ }^{2-4}$.

Pero las consecuencias de nacer con pesos muy bajos también se presentan para sus familias, ya que ellas deben proveerles cuidados especiales y, muchas veces, costosos y los servicios de salud deben brindarles atención altamente calificada no tan solo durante sus primeras horas de vida sino durante sus etapas posteriores de crecimiento debido a los problemas antes descritos ${ }^{5,6}$.

Debido a lo anterior se hace necesario poder estimar los factores de riesgo asociados a estos nacimientos con muy bajo peso, ya que sería factible intervenir precozmente y así ayudar a evitar que se produzcan.

Por esta razón se desarrolló esta investigación que consideró a todos los recién nacidos con pesos inferiores a 2.500 gramos, de las cohortes de nacimientos de los años 2001 y 2002, de la Maternidad del Hospital 
Clínico de la Universidad de Chile, y cuyo objetivo es determinar los factores de riesgo de nacer con muy bajo peso y sus riesgos atribuibles.

\section{MATERIAL Y MÉTODO}

Estudio de corte transversal que consideró a la totalidad de los niños nacidos vivos con peso menor a 2.500 gramos $(n=334)$, en la Maternidad del Hospital Clínico de la Universidad de Chile durante los años 2001 y 2002. Este grupo fue comparado con aquellos que poseían muy bajo peso (peso inferior a 2.000 gramos) y los que pesaron 2.000 gramos y más.

La recolección de la información fue realizada por profesionales en el momento de ocurrencia de la atención prenatal a la embarazada y del parto, debido a que esta maternidad cuenta con un registro electrónico de todas las atenciones brindadas.

El análisis estadístico se dividió en tres fases: la primera consideró una evaluación univariada de los posibles factores asociados al riesgo de nacer con peso inferior a 2.000 gramos. Posteriormente, se construyeron modelos de regresión logística de máxima verosimilitud para identificar los factores de riesgo de nacer con muy bajo peso. En los modelos de regresión se evaluó interacción entre las variables y se verificó la distribución de los residuos. Como no se encontró la existencia de interacción, en la construcción del modelo final se realizó un modelo máximo que incluía todas las variables estudiadas y luego mediante stepwise, se procedió a eliminar aquellas variables cuyo $\mathrm{p}$ era $\geq \mathrm{a} 0,05$ tanto para su ingreso como egreso al modelo.

Una vez identificados los factores de riesgo de nacer con muy bajo peso, se realizó la estimación de la fracción atribuible (FA) de acuerdo a la fórmula?:

$$
\mathrm{FA}=(\mathrm{OR}-1) / \mathrm{OR}
$$

El análisis de la información incluyó la evaluación de la calidad y consistencia de los datos. El análisis y modelaje estadísticos fueron realizados en el programa Stata 8.0 .

\section{RESULTADOS}

En la Tabla 1 se puede observar que los niños seleccionados presentan en promedio un peso y estatura muy bajo y, si bien, sólo el $11 \%$ de los niños es prematuro, un tercio de los recién nacidos de término tuvo como diagnóstico restricción de crecimiento intrauterino (RCIU), como se observa en la Tabla 2.

Entre las patología más frecuentes en la madre se encuentra la preeclampsia y rotura prematura de membranas y en los niños, la restricción de crecimiento intrauterino (Tabla 2 ).

Al analizar la fuerza de asociación en forma univariada, se puede observar que los mayores riesgo de pesar menos de 2.000 $g$ al nacer están relacionados con prematuridad, antecedente de preeclampsia y edades maternas extremas (Tabla 3 ).

En la Tabla 4 se puede observar que en el modelo final, permanecieron las mismas variables que en el análisis univariado habían sido significativas, resaltando que la fuerza de asociación observada en la edad gestacional y edades maternas extremas es mayor que la tabla anterior.

En la Tabla 5 se puede observar que la mayor fracción atribuible está representada por la prematurez, que explica casi el $90 \%$ de los nacimientos de muy bajo peso, seguida de las edades extremas de la madre con un $50 \%$ del riesgo atribuible, $y$, finalmente, la preeclampsia con poco más del $40 \%$.

Tabla 1. Características generales de los recién nacidos

\begin{tabular}{|c|c|c|c|}
\hline Característica & & Promedio & Desviación estándar \\
\hline Peso al nacer $(\mathrm{g})$ & & 1.783 & \pm 629 \\
\hline Longitud al nacer $(\mathrm{cm})$ & & 41,8 & $\pm 5,5$ \\
\hline Índice de masculinidad & $50,9 \%$ & & \\
\hline Índice de prematuridad & $10,8 \%$ & & \\
\hline
\end{tabular}


Tabla 2. Antecedentes de patologías de madres e hijos

\begin{tabular}{lc}
\hline Patología & Frecuencia $(\%)$ \\
De la madre & 29,04 \\
Preeclampsia & 22,29 \\
Rotura prematura de membrana & 14,67 \\
Infección durante el embarazo & 6,61 \\
Hipertensión arterial previa al embarazo & 2,83 \\
Diabetes gestaciónal & 2,69 \\
Tabaquismo durante el embarazo & 30,56 \\
Del niño & 8,2 \\
Restricción de crecimiento intrauterino & \\
Malformaciones &
\end{tabular}

Tabla 3. Estimación puntual e intervalo de confianza (IC) de 95\% de la razón de disparidad (odds ratio, $\psi$ ) de bajo peso al nacer (BPN)

\begin{tabular}{|c|c|c|c|}
\hline Característica & $\Psi$ & Valor p & IC $95 \%$ para $\psi$ \\
\hline Edad Gestacional $\leq 37$ semanas & 5,0 & 0,000 & 2,0 a 12,5 \\
\hline Intervalo Intergenésico previo $\leq 2$ años & 1,20 & 0,406 & 0,77 a 1,88 \\
\hline Gestación previa desfavorable & 0,64 & 0,063 & 0,41 a 1,02 \\
\hline Estatura matema $\leq 1,5 \mathrm{~m}$ & 1,66 & 0,145 & 0,83 a 3,29 \\
\hline Preeclampsia & 1,87 & 0,010 & 1,15 a 3,01 \\
\hline Tabaquismo ( $>$ a $10 \mathrm{cig}$ ) & 0,91 & 0,890 & 0,24 a 3,45 \\
\hline Edad Materna $\geq 3506 \leq 21$ & 1,78 & 0,014 & 1,12 a 2,83 \\
\hline Menos de 6 controles prenatales & 1,00 & 0,997 & 0,64 a 1,56 \\
\hline Más de 3 gestaciones previas & 0,82 & 0,547 & 0,43 a 1,55 \\
\hline HTA crónica & 0,78 & 0,584 & 0,32 a 1,88 \\
\hline Escolaridad materna básica & 0,72 & 0,507 & 0,28 a 1,85 \\
\hline Escolaridad materna media & 1,07 & 0,759 & 0,67 a 1,71 \\
\hline
\end{tabular}

Tabla 4. Factores de riesgo de peso muy bajo al nacer $(<2.000 \mathrm{~g})$

\begin{tabular}{lrrr}
\hline Factor de Riesgo & $\psi$ & Valor p & IC 95\% para $\psi$ \\
\hline Edad Gestacional $\leq 37$ semanas & 9,11 & $<0,0001$ & $2,66 \mathrm{a} 31,12$ \\
Preclampsia & 1,80 & 0,027 & $1,06 \mathrm{a} 3,05$ \\
Edad Materna $\leq 350621$ & 2,03 & 0,007 & $1,21 \mathrm{a} 3,39$ \\
$\mathrm{X}^{2}=32,52$, con $3 \mathrm{gl}$ & & &
\end{tabular}

Tabla 5. Fracciones atribuibles de prematurez, ser hijos de madres con edades extremas y antecedente de preeclampsia

\begin{tabular}{lrrr}
\hline Característica & Fracción atribuible & Valor p & Intervalo de confianza \\
\hline Edad Gestacional $\leq 37$ semanas & $89,0 \%$ & $<0,0001$ & $62,4 \%$ a $96,7 \%$ \\
Edad Materna $2350 \leq 21$ & $50,7 \%$ & 0,007 & $17,3 \%$ a $70,5 \%$ \\
Pre eclampsia & $44,5 \%$ & 0,027 & $5,6 \%$ a $67,2 \%$
\end{tabular}




\section{DISCUSIÓN}

Este trabajo permite concluir que los factores de riesgo de muy bajo peso al nacer han cambiado en el transcurso de una década, ya que un estudio realizado en un hospital de la zona oriente de la capital demostró que los factores nutricionales maternos eran en esa época, los que estaban fuerte y únicamente asociados a esta condición ${ }^{8}$.

Este cambio en los factores de riesgo estaría explicado por un cambio en el perfil epidemiológico que la población chilena experimentó en estos últimos años, transformando los problemas de malnutrición por déficit por los de exceso ${ }^{9}$ y debido a que durante décadas, los programas ministeriales focalizaron sus esfuerzos hacia las poblaciones en riesgo, especialmente nutricional, disminuyendo en forma dramática la malnutrición por déficit ${ }^{10}$.

En este trabajo se plantea que el factor de riesgo mayormente asociado a muy bajo peso al nacer es la prematuridad, lo cual ha sido reportado en estudios llevados a cabo en paises desarrollados". Que un niño de pretérmino nazca con menor peso es coherente y plausible biológicamente, ya que se ha estimado que por cada semana que el niño está dentro del útero gana en promedio entre 200 y $300 \mathrm{~g}$, especialmente hacia el final del embarazo ${ }^{12}$.

La segunda causa observada es la edad materna extrema. $\mathrm{Al}$ respecto, se ha planteado que las mujeres adolescentes tendrían un mayor riesgo a tener partos prematuros o a que sus hijos de término nazcan con menor peso debido a que presentan un estado nutricional pre concepcional deficitario, tienen una escasa ganancia de peso durante el embarazo asisten escasamente a controles e, incluso, pueden llegar a tener desórdenes hipertensivos.

En la mujeres con edades mayores a 35 años, se ha planteado que la explicación sería la existencia de enfermedades crónicas como diabetes e hipertensión y placenta previa, lo que traería como consecuencia adelantar la fecha del parto ${ }^{13-15}$.

La preeclampsia ya ha sido señalada en la literatura como un factor de riesgo fuertemente asociada al bajo peso al nacer ${ }^{16,17}$. Entre las explicaciones está que en los casos en que la madre presenta preeclampsia y los niños nacen pre término, este hecho estaría mediado por una anormalidad placentaria, en cambio cuando el niño es de término, se ha señalado que podrían haber una mezcla de condiciones que incluirían afecciones placentarias o condiciones hipertensivas en el embarazo sin disfunción placentarials.

El hecho que las fracciones atribuibles asociadas a los tres factores de riesgo antes mencionados sean altas, hace pensar que se podrían realizar esfuerzos para prevenir su aparición y así disminuir el porcentaje de niños con pesos muy bajos al nacer. No obstante esta premisa, habría que plantear si es posible realizar acciones tendientes a disminuir los partos de pretérmino, embarazos en edades extremas y preeclampsia.

\section{REFERENCIAS}

1.- AMIGO H, BUSTOS P, ZUMELZU E. Secular changes in anthropometrical variables at birth: a local perspective. Rev Méd Chile 2002; 130: 1095100 .

2.- WEINDRICH D, JENNEN-STEINMETZ C, LAUCHT M, SCHMIDT M H. Late sequelae of low birthweight: mediators of poor school performance at 11 years. Dev Med Child Neurol 2003; 45: 463-9.

3. PINTO-MARTIN J, WHITAKER A, FELDMAN J, CNAAN A, ZHAO H, ROSEN-BLOCH J et al. Special education services and school performance in a regional cohort of low-birthweight infants at age nine. Paediatr Perinat Epidemiol 2004; 18: 1209.

4.- BAZAES R A, SALAZAR T E, PlTTALUGA E, PENA V, ALEGRIA A, INIGUEZ G et al. Glucose and lipid metabolism in small for gestational age infants at 48 hours of age. Pediatrics $2003 ; 111(4 \mathrm{Pt}$ 1): $804-9$.

5.- LANDRY S H, SMITH K E, SWANK P R. The importance of parenting during early childhood for school-age development. Dev Neuropsychol 2003; 24(2-3): $559-91$.

6.- MOORE K A, COKER K, DUBUISSON A B, SWETT B, EDWARDS W H. Implementing potentially better practices for improving familycentered care in neonatal intensive care units: successes and challenges. Pediatrics 2003; 111 (4 Pt 2): $450-60$.

7.- KURITZ S J, LANDIS J R. Summary attributable risk estimation from unmatched case-control data. Stat Med 1988; 7(4): 507.17.

8.- VEGA J, SAEZ G, SMITH M, AGURTO M, MORRIS N M. Risk factors for low birth weight 
and intrauterine growth retardation in Santiago, Chile. Rev Méd Chile 1993; 121: 1210-9.

9.- ALBALA C, VIO F, KAIN J, UAUY R. Nutrition transition in Chile: determinants and consequences. Public Health Nutr 2002; 5( I A): 123-8.

10.- UAUY-DAGACH R. The practice of clinical nutrition in a developing nation. J Nutr 1994; $124(8$ Suppl): 1449S-54S.

11.- WEN S W, KRAMER M S, PLATI R, DEMISSIE K, JOSEPH K S, LIU S et al. Secular trends of fetal growth in Canada, 1981 to 1997. Paediatr Perinat Epidemiol 2003; 17: 347-54.

12.- JUEZ G, VENTURA-JUNCA DEL TOBAR P, LUCERO E F. Intrauterine growth in a selected group of Chilean newborn infants. Subdiagnosis of intrauterine growth retardation in Chile? Rev Méd Chile 1984; 112: 759-64.

13.- OZALP S, METE TANIR H, SENER T, YAZAN S, KESKIN A E. Health risks for early $(<$ or $=19)$ and late $(>$ or $=35$ ) childbearing. Arch Gynecol Obstet 2003; 268: 172-4.

14.- JOLLY M, SEBIRE N, HARRIS J, ROBINSON S, REGAN L. The risks associated with pregnancy in women aged 35 years or older. Hum Reprod 2000; 15: $2433-7$.

15.- CHANG S C, O'BRIEN K O, NATHANSON M S, MANCINI J, WITTER F R. Characteristics and risk factors for adverse birth outcomes in pregnant black adolescents. J Pediatr 2003; 143: 250-7.

16.- XIAO R, SORENSEN T K, WILLIAMS M A, LUTHY D A. Influence of pre-eclampsia on fetal growth. J Matern Fetal Neonatal Med 2003; 13: $157-62$.

17.- RASMUSSEN S, IRGENS L M. Fetal growth and body proportion in preeclampsia. Obstet Gynecol $2003 ; 101: 575-83$.

18.- VATTEN L J, SKJAERVEN R. Is pre-eclampsia more than one disease? BJOG 2004; 111: 298-302.

Usted puede comentar éste y otros artículos publicados en la Revista Chilena de Salud Pública, enviando un correo electrónico a revistasp@med.uchile.cl 